\title{
A Review on Different Routing Protocols on Wireless Sensor Network
}

\author{
Archana Jangra \\ BITS, BHIWANI \\ Department of Computer Science
}

\begin{abstract}
Wireless sensor networks are a collection of small devices known as sensors. These Sensor nodes have some limited resources such as cache, strength, and potential. To design a network every sensor network should be connected to each sensor so that the communication can take place between every node. This communication process consumes lots of energy. So it is necessary to develop a powerful and energy efficient protocol to increase the lifespan of the whole network. These protocols can work as routers and also reliable in multi-hop communication. This paper will give a brief introduction on various routing protocol such as hierarchical, location based and data-centric. It will also give a review on advantage, disadvantages, limitations and strengths.
\end{abstract}

\section{Keywords}

WSN, Energy, Sensor Node, Lifetime, Communication

\section{INTRODUCTION}

WSN is now booming itself as a very powerful tool in communication. Earlier, it was used by some big academia and industries only. In sensor networks, energy is a critical resource because network's whole functioning totally depends on the battery. So there is a need to improve the energy efficiency. This requirement of sensor node network made their architecture and protocol challenging.

A sensor network is a collection of small devices called nodes. The basic component of the network is a sensor which is essential for monitoring physical conditions such as humidity, pressure, motion, sound, temperature etc. These sensor nodes collect data from their surrounding environment. To calculate routes in the network, the network layer is used. There can be multiple paths between source and destination.

\section{WSN DESIGN ISSUES}

Simplicity, adaptability, energy efficient and scalability are some important characteristics of wireless sensor network. The main challenge in front of WSN is to prolong the lifetime of the network.

The construction of routing protocol in WSNs is influenced by some important parameters as discussed below.

\subsection{Node Deployment}

Deployment of a node can be either manual or random. In manual deployment, the node can be manually placed and data routed through some selected paths. Whereas in random system nodes are scattered randomly creating an infrastructure in an ad hoc network. In random system distribution of node is non-uniform.

\subsection{Network Dynamics}

Most applications depend on the immobile sensors so most of the architectures assume this system is made only for the stationary sensing system. But in few applications moving sensor nodes can be more effective rather than stationary. So Communication through moving node is more challenging in terms of bandwidth and energy.

\subsection{Energy Conservation}

To elect the better route during communication is totally depends on the energy level of the system. Since the transmission power of a wireless radio is proportional to distance squared or even higher order in the presence of obstacles, multi-hop routing will consume less energy than direct communication.

\subsection{Scalability}

The device should be made reliable to handle the thousands of targets.

\subsection{Hardware Constraints}

All devices under the sensor network should consume small power so that the lifetime of the network should be maximized.

\subsection{Environment}

Sensor networks are mostly useful in a hostile environment so the device should be environmental friendly.

\subsection{Transmission media}

In WSN transmission media is generally RF or infrared which is mostly affected by fading.

\subsection{Routing techniques of WSN}

Routing techniques are classified into seven categories. These techniques are divided on the basis of network structure, operation etc but in this paper, only location based protocols and hierarchical protocols are discussed.

\begin{tabular}{|l|l|}
\hline \multicolumn{1}{|c|}{ Category } & \multicolumn{1}{c|}{ Representative Protocols } \\
\hline Location-based Protocols & MECN, SMECN, GAF, GEAR, Span, TBF, BVGF, GeRaF \\
\hline Data-centric Protocols & $\begin{array}{l}\text { SPIN, Directed Diffusion, Rumor Routing, COUGAR, } \\
\text { ACQUIRE, EAD, Information-Directed Routing, Gradient- } \\
\text { Based Routing, Energy-aware Routing, Information-Directed } \\
\text { Routing, Quorum-Based Information Dissemination, Home } \\
\text { Agent Based Information Dissemination }\end{array}$ \\
\hline Hierarchical Protocols & LEACH, PEGASIS, HEED, TEEN, APTEEN \\
\hline Mobility-based Protocols & $\begin{array}{l}\text { SEAD, TTDD, Joint Mobility and Routing, Data MULES, } \\
\text { Dynamic Proxy Tree-Base Data Dissemination }\end{array}$ \\
\hline Multipath-based Protocols & $\begin{array}{l}\text { Sensor-Disjoint Multipath, Braided Multipath, N-to-1 } \\
\text { Multipath Discovery }\end{array}$ \\
\hline Heterogeneity-based Protocols & IDSQ, CADR, CHR \\
\hline QoS-based protocols & SAR, SPEED, Energy-aware routing \\
\hline
\end{tabular}




\section{DESCRIPTION OF LOCATION BASED PROTOCOLS}

In this protocol, nodes are known by their location. In this part, only location based protocols made for WSN are discussed.

\subsection{Graphical adaptive fidelity (GAF)}

This is energy-aware protocol basically designed for MANETs, but also can work for wireless sensor networks because it also has the capability to save the energy. GAF is designed so that it can calculate energy consumption during transmission and reception. GAF has the capability to turn off the unnecessary sensor nodes. In GAF sensor field is divided into grid squares and each node uses its location information.

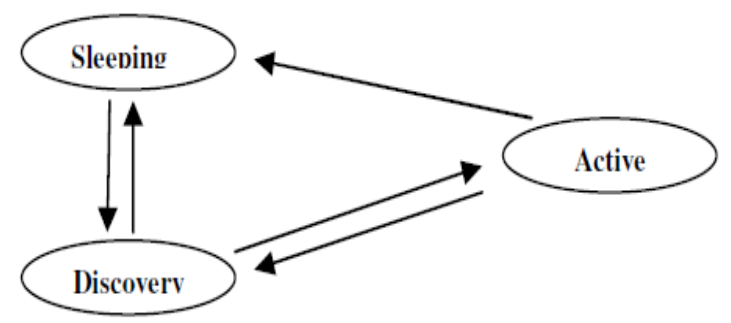

Figure 1: State transition diagram of GAF

The diagram shows GAF has three states such as Sleeping, Active, and Discovery.

\subsection{Geographic and Energy Aware Routing (GEAR)}

It is made to target region in the node field. In GEAR, every node is equipped with local hardware. For instance, a GPS system is used to know the current position. Moreover, every sensor has information of residual energy and about their location.

\subsection{Coordination of Power Saving with Routing}

This protocol is also made for MANETs, but also helpful in wireless sensor network. This protocol has the capability to turn off the RADIO during idle time. Span does not provide any kind of location information. Span made good coordination between the nodes. So it very easy to transmit packets from source to destination. It uses geographical forwarding protocol to transmit the packet. In SPAN election based on the advertisement send by each node to their neighbors about their status.

\subsection{Trajectory -based forwarding (TBF)}

TBF needs very complex network and the presence of coordinator system. For example, a GPS, so that sensor can sense the location and distance between the neighbor nodes. Source specifies the trajectory in the packet. The decision of path in TBF is not affected by node mobility. To increase the reliability and the capacity multipath routing is also needed in TBF. This protocol also can be used for functions such as flooding, Discovery, and network management.

\subsection{Bounded Voronoi Greedy Forwarding (BVGF)}

In this, every sensor has the information of their geographical location. In this approach, a sensor will always send a packet to a neighbor that has smallest Euclidean distance from the destination. In BVGF every sensor has only one next hop to send its packets so every route between source ad sink will always have the same chain of next hop.

\subsection{Minimum energy communication network (MECN)}

It is location based protocol. Sensors are deployed randomly. MECN is self-configurable protocol. MECN uses minimum power topology. This approach considers minimum power path from sensor to sink. Every node sends a message to its neighbor about its cost. Where the cost of a node is minimum power required to select the path. MECN is also a faulttolerant protocol

\subsection{Small minimum energy communication network (SMECN)}

This protocol is an improvement of MECN, in which a minimal graph is characterized with regard to the minimum energy property. This property implies that for any pair of sensors in a graph associated with a network, there is a minimum energy-efficient path between them.

In SMECN each node computes its neighbor by advertising a message with help of initial power. This neighbor has computed analytically.

\section{DESCRIPTION OF VARIOUS HIERARCHICAL ROUTING PROTOCOLS}

\subsection{PEGASIS}

PEGASIS stands for Power-Efficient Gathering in Sensor Information Systems. This protocol is optimization of LEACH protocol. The idea behind this protocol is to make a chain of sensor nodes so that every 111 nodes can receive information from the transmitter and directly connected with neighbors. Gathered data move from the first node to last node. When one node dies in this chain then the chain will be reconstructed. PEGASIS collects data from round to round.

Features of PEGASIS

1. PEGASIS do not use cluster formation because greedy approach made it more feasible.

2. The distance between the neighboring nodes is very small as compared to cluster head node.

3. PEGASIS added excessive delay for a distant node on the chain.

\subsection{Threshold Sensitive Energy Efficient Sensor Network Protocol (TEEN)}

This is a hierarchical clustering protocol, all sensor nodes are grouped into a single cluster. A sensor node controls the whole network is known as a cluster head. Every node sends its data to the cluster head. Teen uses data-centric approach. It is very useful where data accuracy is required. However TEEN is not useful for sensing applications where the periodic report is needed.

\subsection{Adaptive Periodic Threshold Sensitive Energy Efficient Sensor Network Protocol (APTEEN):}

It is an updated protocol of TEEN to reduce shortcomings and time critical events. It is hybrid clustering based protocol. Architecture of both APTEEN and TEEN is same. APTEEN sense data periodically which means it reacts on sudden change in sensed value. 
APTEEN works on three different types of queries such as:-

1) Persistent query, to sense an event in a given period of time.

2) One time query, it clicks a photograph or snapshot of the network.

3) Historical query, to verify or to analyze past data

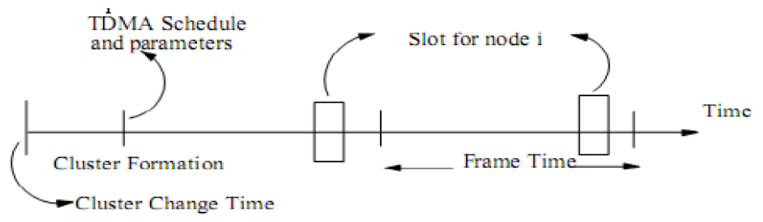

Figure 2:operation of APTEEN

Advantages of APTEEN

1) Energy consumption is less.

2) Ensures that large number of sensing node will be in working condition

3) According to the demonstrations it has been concluded that the energy dissipation of APTEEN is between LEACH and TEEN

Disadvantages of APTEEN

1) Cluster formation is complex

2) Implementing threshold based function

\subsection{Low Energy Adaptive Clustering Hierarchy (LEACH)}

To distribute the whole load of network evenly LEACH uses randomized rotation. All nodes are connected with a cluster head and each node sends data to the cluster head. After data aggregation, $\mathrm{CH}$ sends this data to base station. There are three main features of LEACH such as:-
1) Control of cluster set-up
2) Randomized rotation of $\mathrm{CH}$
3) Compression to reduce global data

Load on the $\mathrm{CH}$ is more so it is important to give powerful resources to $\mathrm{CH}$. The main function of $\mathrm{Ch}$ is to collect data from all sub-nodes and then compress data with help of some compression algorithms and the send compressed data to the BS. Compression is done to save the energy. Here data collection is centralized and periodic. When stable sensing is required this protocol is proved itself as the best protocol.

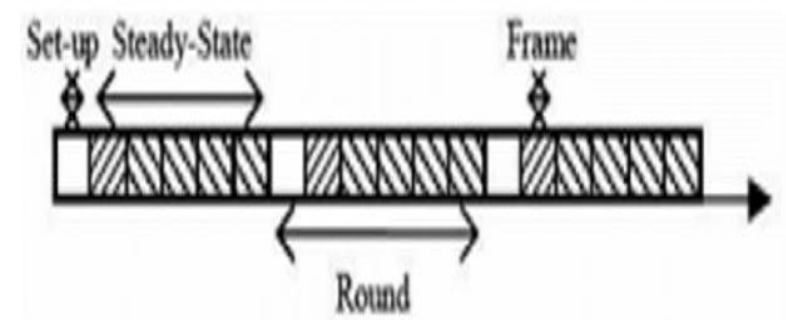

Figure 3:Two phases of LEACH protocol

LEACH works in two phases.

1) Set-up phases

2) Steady state phase
In set-up phase, set-up of the cluster is done and $\mathrm{CH}$ selected by the members of the cluster. In second phase actual data is transferred to the BS. Set-up phase takes less time than the steady state phase. During the setup phase, a sensor node selects a random number between 0 and 1 . If this selected value is less than the threshold value $\mathrm{T}(\mathrm{n})$, the node becomes $\mathrm{CH}$ for the current round. The threshold value is decided by the equation written below.

$$
T(n)=\left\{\begin{array}{cc}
\frac{p}{1-p * r \bmod \left(\frac{1}{p}\right)} & \text { if } n \in G \\
0 \quad \text { otherwise }
\end{array}\right.
$$

Where

$\mathrm{T}(\mathrm{n})$ - Threshold value

$\mathrm{r}$ - Current round

$\mathrm{p}$ - Predetermined fraction of node

\subsection{Adaptive LEACH (A-LEACH)}

According to this protocol, cluster head consumes lots of energy to compare other nodes in the network. It is clustering based protocol. Adaptive LEACH proposed a new algorithm to select the cluster head. This protocol added some new parameter in threshold equation of LEACH. These parameters are the general probability $(\mathrm{Gp})$ and current status probability (CSp).

$$
\begin{gathered}
T(n)-C_{p} \mid C S_{p} \\
-\frac{k}{\left(N-k\left(r \bmod \frac{N}{k}\right)\right.}+\frac{E_{\text {Current }}}{E_{n-\max }} \\
* \frac{K}{N}
\end{gathered}
$$

Where

$\mathrm{K}$ - Expected number of cluster head in a round

$\mathrm{N}$ - Total number of nodes in the network

$\mathrm{r}$ - Current round

\subsection{Hybrid Energy Efficient Distributed clustering (HEED)}

HEED is modified protocol of LEACH by using residual energy in a multi-hop network. HEED uses adaptive power communication. This protocol has four aims such as:-

1) With help of distributed energy consumption to increase the network lifetime.

2) In fixed number of irritations terminate the clustering process.

3) To reduce control and overhead

4) Select well-distributed cluster head

In HEED, $\mathrm{CH}$ is selected periodically based on two parameters. The first parameter is residual energy and the second parameter is intra-cluster communication cost. HEED have better energy efficiency than LEACH because to select 
$\mathrm{CH}$ in LEACH random process is used. These parameters are sufficient to improve the network lifetime in HEED.

\section{CONCLUSION AND FUTURE RESEARCH}

One of main challenge in front of WSN is to design energy efficient network. Most of the energy is consumed in transmission and reception. So it is necessary to design a protocol that can prolong the lifetime of the whole network.

In this paper, various routing protocols depend on their capabilities and parameters have been reviewed. This will give an opportunity for researchers to do some improvements in the basic protocols so that a suitable and energy efficient protocol can be designed.

\section{REFERENCES}

[1].Vinay Kumar, Sanjeev Jain and SudarshanTiwari , “ Energy Efficient Clustering Algorithms in Wireless Sensor Networks: A Survey" , IJCSI International Journal of Computer Science Issues, Vol. 8, Issue 5, No 2, September 2011

[2].Minhas, A.A.; Trathnigg, T.; Steger, C.; Weiss, R., "Energy saving in pervasive wireless sensor networks", Intelligent Environments, 2006. IE 06. 2nd IET International Conference Volume: 1 Publication Year: 2006 , Page(s): $381-389$

[3].Zaballos, A.; Vallejo, A.; Terradellas, P.; Selga, J.M.,”A Genetic-Based QoS Aware Routing for Ubiquitous Sensor Network",Wireless and Mobile Computing, Networking and Communications, 2009. WIMOB 2009. IEEE International Conference on 2009 , Page(s): 129 134.

[4].Sarjoun S. Doumit, Dharma P. Agrawal: Self-Organizing and Energy-Efficient Network of Sensors, IEEE, pp. 1-6 (2002).

[5].Prabhat Kumar and M. P. Singh , "A New Clustering Protocol Based on Energy Band for Wireless Sensor Network", International Journal of Information Technology and Knowledge Management, Volume 3,January -June 2010, ISSN :0973-4414

[6].W. Heinzelman, J. Kulik, and H. Balakrishnan, "Adaptive Protocols for Information Dissemination in Wireless Sensor Networks," Proc. 5th ACM/IEEE Mobicom Conference (MobiCom '99), Seattle, WA, August, 1999. pp. 174-85.

[7].I. Akyildiz, W. Su, Y. Sankarasubramaniam, and E. Cayirci, "A survey on sensor networks," IEEE Communications Magazine, Volume: 40 Issue: 8, pp.102-114, August 2002.

[8].Thomas Watteyne, AntonellaMolinaro, Maria GraziaRichichi and MischaDohler, "From MANET To IETF ROLL Standardization: A Paradigm Shift in WSN Routing Protocols", IEEE COMMUNICATIONS SURVEYS \& TUTORIALS, VOL. 13, NO. 4, FOURTH QUARTER 2011, pp. 688-707

[9].M. P. Singh and M. M. Gore, A new energy-efficient clustering protocol for wireless sensor networks In Proceedings of the Second International Conference on Intelligent Sensors, Sensor Networks and Information Processing (ISSNIP 2005) (Melbourn, Australia, dec 2005), pp. 25-30.
[10].I. F. Akyildiz and W. Su and Y. Sankarasubramaniam and E. A. Cayirci A survey on sensor network, IEEE Communication Magazine 40, 8 (August 2004), 102-114.

[11].V. Rodoplu and T.H. Ming, "Minimum energy mobile wireless networks," IEEE Journal of Selected Areas in Communications, Vol. 17, No. 8, 1999,pp.1333-1344.

[12]. W. R. Heinzelman and A. Chandrakasan and H. Balakrishnan, Energy-efficient communication protocol for wireless microsensornetwork,HICSS, 2000.

[13]. Rongxin Li, ChaomeiZheng, Yunru Zhang , "Study of Power-Aware Routing Protocol in Wireless Sensor Networks" 2011 IEEE

[14]. Mark Hempstead, Michael J. Lyons, David Brooks, and Gu-Yeon Wei, "Survey of Hardware Systems for Wireless Sensor Networks " Journal of Low Power Electronics Vol.4, 1-10, 2008

[15].Ali Norouzi and Abdul HalimZaim, "An Integrative Comparison of Energy Efficient Routing Protocols in Wireless Sensor Network", Wireless Sensor Network, 2012, 4, 65-75

[16]. Mohammad Reza Mazaheri, BehzadHomayounfar and SayyedMajidMazinani "QoS Based and Energy Aware Multi-Path Hierarchical Routing Algorithm in WSNs" Wireless Sensor Network, 2012, 4, 31-39, http://www.scirp.org/journal/wsn

[17]. Lindsey, S.; Raghavendra, C.S. PEGASIS: PowerEfficient Gathering in Sensor Information Systems. In Proceedings of the Aerospace Conference, Big Sky, MT, March, 2002; pp. 1125-1130.

[18].A. Manjeshwar and D. P. Agarwal, "TEEN: a Routing Protocol for Enhanced Efficiency in Wireless Sensor Networks," 1st Int"1. Wksp. on Parallel and Distrib. Comp. Issues in Wireless Networks and Mobile Comp., April 2001. [19]. A. Manjeshwar and D. P. Agarwal, "APTEEN: A Hybrid Protocol for Efficient Routing and Comprehensive Information Retrieval in Wireless Sensor Networks," Proc. Int" 1 . Parallel and Distrib. Proc. Symp., pp. 195-202.

[20]. W. Heinzelman, A. Chandrakasan, and H. Balakrishnan: Energy-efficient communication protocol for wireless sensor networks, in the Proceeding of the Hawaii International Conference System Sciences, Hawaii ( January 2000).

[21]. S.K. Singh, M.P. Singh, and D.K. Singh, "A survey of Energy-Efficient Hierarchical Cluster-basedRouting in Wireless Sensor Networks", International Journal of Advanced Networking andApplication (IJANA), Sept.Oct. 2010, vol. 02, issue 02, pp. 570-580.

[22]. S.K. Singh, M.P. Singh, and D.K. Singh, "Energyefficient Homogeneous Clustering Algorithm forWireless Sensor Network", International Journal of Wireless \& Mobile Networks (IJWMN), Aug.2010, vol. 2, no. 3, pp. 49-61.

[23].Jun Zheng and Abbas Jamalipour, "Wireless Sensor Networks: A Networking Perspective", a book published by A John \& Sons, Inc, and IEEEE, 2009.

[24].S. Misra et al. (eds.), Guide to Wireless Sensor Networks, Computer Communications and Networks, 
International Journal of Computer Applications (0975 - 8887)

Volume 149 - No.12, September 2016

DOI: $\quad 10.1007 / 978-1-84882-218-4 \quad 4$, Springer-Verlag London Limited 2009.

[25].Ivan Stojmenovic and Stephan Olariu. Data-centric protocols for wireless sensor networks. InHandbook of Sensor Networks, Chapter 13, pages 417-456. Wiley, 2005.

[26].Christopher Ho, Katia Obraczka, Gene Tsudik, and Kumar Viswanath, "Flooding for reliable multicast in multi-hop ad hoc networks", In Proceedings of the 3rd International Workshop on DiscreteAlgorithms and
Methods for Mobile Computing and Communications (DIAL-M'99), 1999, pp. 64-71.

[27].Ming Liu, Jiannong Cao, Guihai Chen, and Xiaomin Wang, "An Energy-Aware Routing Protocol in Wireless Sensor Networks", Sensors 2009, vol. 9, pp. 445-462.

[28] Luis Javier García Villalba, Ana Lucila Sandoval Orozco, Alicia Triviño Cabrera, and Cláudia Jacy Barenco Abbas, "Routing Protocol in Wireless Sensor Networks", Sensors 2009 